\title{
Vector Calibration for Vertical Tests of Superconducting RF Cavities
}

\author{
Daniil Frolov
}

Fermilab, Batavia, IL 60510

November 2019

\begin{abstract}
We propose procedure for vector calibration of $Q_{0}$ vs $E_{a c c}$ curve measurement for superconducting resonators. The proposed vector calibration procedure and hardware has several advantages over the techniques currently used for vertical cavity tests. It provides high level of automation and reduces manpower and preparation time for the test; it provides in situ, real time control of hardware parameters and allows to detect hardware failure and operator's errors; it allows to control and exclude the most significant systematic uncertainties, measured values of $Q_{0}\left(E_{a c c}\right)$ can be made traceable to national standards.
\end{abstract}

\section{Introduction}

Vertical testing of SRF cavities in a helium bath is used to measure their main parameters: intrinsic quality factor and accelerating gradient. Practical developments in material science, aimed to improve cavity performance heavily rely on these measurements, therefore it is critically important to ensure their accuracy and repeatability.

Determination of intrinsic quality factor and accelerating gradient is based on the analysis of the r.f. power that is reflected from the cavity, dissipated in the cavity and transmitted through it. These measurements can contain significant errors if features of r.f. networks with distributed parameters are not taken into account. To author's knowledge all known operational SRF facilities are using methods of scalar network analysis (based only on power measurements) for vertical test stand (VTS) calibration, however these methods are approximate by definition. In this report we propose to use methods of vector network analysis (based on magnitude and phase measurements) for a complete VTS calibration. It is important to mention that some ideas of VTS measurements with elements of vector correction were proposed earlier. In [2] authors used variable delay line installed between cavity and incident power source, similar to how it was proposed in 1977 for network analyzers based on six-port reflectometers 
[5]. And in [3] authors used cavity in a self excited loop connected to a commercial vector network analyzer. Unfortunately these previous works do not have complete r.f. system models and only partially use the advantages of vector measurements.

Error models in this report are used in all modern vector network analyzers (VNA) and are standard in the r.f. measurement community (see general error models in [12], [9], [8]). But these known calibration procedures and especially hardware architecture of commercially available VNAs cannot be directly used for VTS measurements because of such features as arbitrary cavity frequency detuning due to helium pressure, high power of test signals and requirement to know absolute power levels rather than ratios. These limitations are analyzed in this paper and improved VTS r.f. system architecture and methods of cavity measurements are proposed. Values of intrinsic quality factor and accelerating gradient measured with these methods can be made traceble to national standards. 


\section{Vertical Test Stand Equations}

For simplicity in this paper we consider an accelerating cavity under test as a two port device: first for incident wave and second for transmitted wave, with no additional ports. For all r.f. measurements VTS facility at Fermilab is using procedure described in [1]. Final cavity parameters intrinsic quality factor $Q_{0}$ and accelerating gradient $E_{a c c}$ are defined in eqs. (1), (2).

$$
\begin{gathered}
Q_{0}=\left(1+\beta_{1}+\beta_{2}\right) \cdot Q_{L}, \\
E_{\text {acc }}=\sqrt{Q_{0} P_{l o s s} \frac{r / Q}{L}}=\left[\frac{V}{m}\right],
\end{gathered}
$$

where:

$\beta_{1}, \beta_{2}-$ coupling of ports 1 and 2 ;

$Q_{L} \quad$ - loaded quality factor of the cavity;

$P_{\text {loss }}$ - loss power in the cavity, $[W]$;

$r / Q \quad$ - geometric factor $[\Omega / m]$;

$L \quad$ - electrical length of the cavity $[m]$;

Among these parameters $Q_{L}$ is usually extracted from the cavity decay time measurement, $r / Q$ and $L$ can be extracted from the electromagnetic simulations. The rest of the parameters $P_{\text {loss }}, \beta_{1}$ and $\beta_{2}$ are extracted form the r.f. measurements (3), (4):

$$
\begin{aligned}
& P_{\text {loss }}=P_{i}-P_{r}-P_{t}, \\
& P_{i}=P_{i m} C_{i}, \\
& P_{r}=P_{r m} C_{r}, \\
& P_{t}=P_{t m} C_{t},
\end{aligned}
$$

where:

$$
\begin{aligned}
& P_{i}, P_{r}, P_{t} \quad-\quad \text { corrected values of incident, reflected } \\
& \text { and transmitted power; } \\
& P_{i m}, P_{r m}, P_{t m} \text { - measured values of power; } \\
& C_{i}, C_{r}, C_{t} \quad-\text { correction coefficients. } \\
& \beta_{2}=P_{t} / P_{\text {loss }}, \\
& \beta_{1}=\beta^{*} \cdot\left(1+\beta_{2}\right) \text {, } \\
& \beta^{*}=\frac{1-\Gamma}{1+\Gamma}, \\
& \Gamma=C_{\beta}|\Gamma| \text {, } \\
& |\Gamma|=\sqrt{P_{r} / P_{i}} \text {, }
\end{aligned}
$$


where $C_{\beta}$ is coupling factor, +1 for under-coupled cavity and -1 for over-coupled and it is selected based on the reflected power envelope shape measured with an oscilloscope.

From eqs. (1) - (4) it can be seen that there are four core measurands that can introduce direct error into $Q_{0}$ and $E_{a c c}$ determination: $Q_{L}, P_{i}, P_{r}, P_{t}$. We shall study last three parameters. According to eq. (3), these parameters are products of measured power and correction coefficients. In VTS r.f. power is measured directly with calibrated power sensors, error of this measurement in general defined by specifications of the sensor. However, error of correction coefficients $C_{i}, C_{r}, C_{t}$ determination is defined by methodology and math that is used for their evaluation. According [1], determination of $C_{i}, C_{r}, C_{t}$ relies on pure scalar corrections, based on direct r.f. power measurements: all signal paths should be characterized separately using signal injection and measurement on the other side of the path. However, with such approach differences in mismatches of various connectors are ignored, matching of the incident and transmitted power port is ignored, electrical length variations in the cables are ignored, and practical implementation of such calibration procedure demands for multiple manual connections and re-connections that requires significant amount of manpower and leads to increased cost of cavity tests and probability of errors. These problems can be particularly severe at high frequencies (at Fermilab SRF cavities up to $9 \mathrm{GHz}$ are tested).

In the following sections instead of measuring scalar ratios of power levels that give only approximate values of $C_{i}, C_{r}, C_{t}$ we propose to determine them based the direct measurements of vector ratios of the scattered waves with full featured vector error correction techniques used in modern r.f. test equipment. We also propose the required new VTS hardware architecture. 


\section{Vertical Test Stand Errors}

\subsection{Error in reflection measurement}

A standard error model for a directional device is described in [7]. Illustration to this model is given in fig. 1. It assumes that between actual reflection coefficient $\Gamma_{A}$ and its measured value $\Gamma_{M}$ there exists an error network that distorts the measured result.
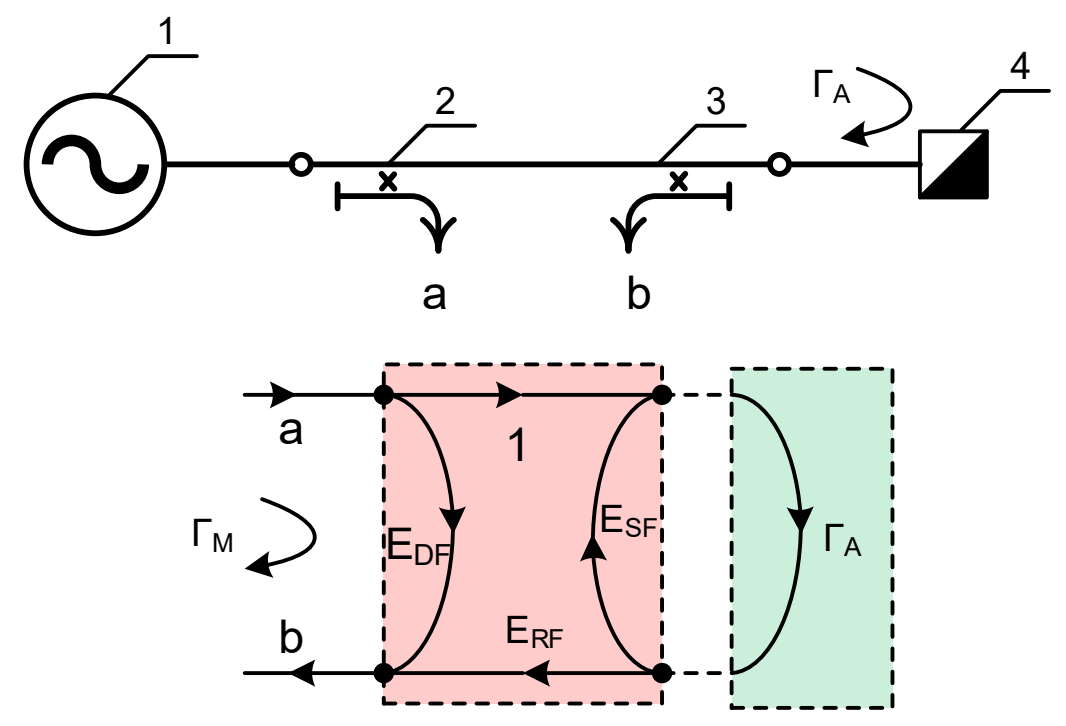

Figure 1: Illustration to reflection error model

1 - r.f. generator; 2 - directional coupler of forward wave; 3 - directional coupler of reflected wave; 4 - load; $\Gamma_{M}, \Gamma_{A}$ - measured and actual values of reflection from load 4;

$E_{D F}$ - directivity, $E_{R F}$ - reflection tracking, $E_{S F}$ - source match.

Under such conditions the measured value of reflection can be found from the flow graph as a ratio $\Gamma_{M}=b / a$. Error in reflection measurement $\delta \Gamma$ can be defined as difference between measured and actual values:

$$
\begin{gathered}
\Gamma_{M}=E_{D F}+\frac{E_{R F} \Gamma_{A}}{1-E_{S F} \Gamma_{A}} \\
\delta \Gamma=\Gamma_{M}-\Gamma_{A}=\frac{E_{R F} \Gamma_{A}-\Gamma_{A}+E_{S F} \Gamma_{A}^{2}}{1-E_{S F} \Gamma_{A}}+E_{D F}
\end{gathered}
$$

Calibration procedure from [1] corrects only for reflection tracking $E_{R F}$ error factor, while the directivity $E_{D F}$ and source match $E_{S F}$ are assumed ideal. It can be seen from the graphs in fig. 2 that under some conditions error can be quite significant. Even if cavity is critically coupled the reflection measurement error can easily reach $100 \%$ if directivity error factor $E_{D F}$ is ignored.

For the real hardware used in Fermilab VTS, directivity is typically $E_{D F}=-30$ $\mathrm{dB}$ or 0.032 defined by the directional coupler (Narda Model 3022 for $1.3 \mathrm{GHz}$ system), 

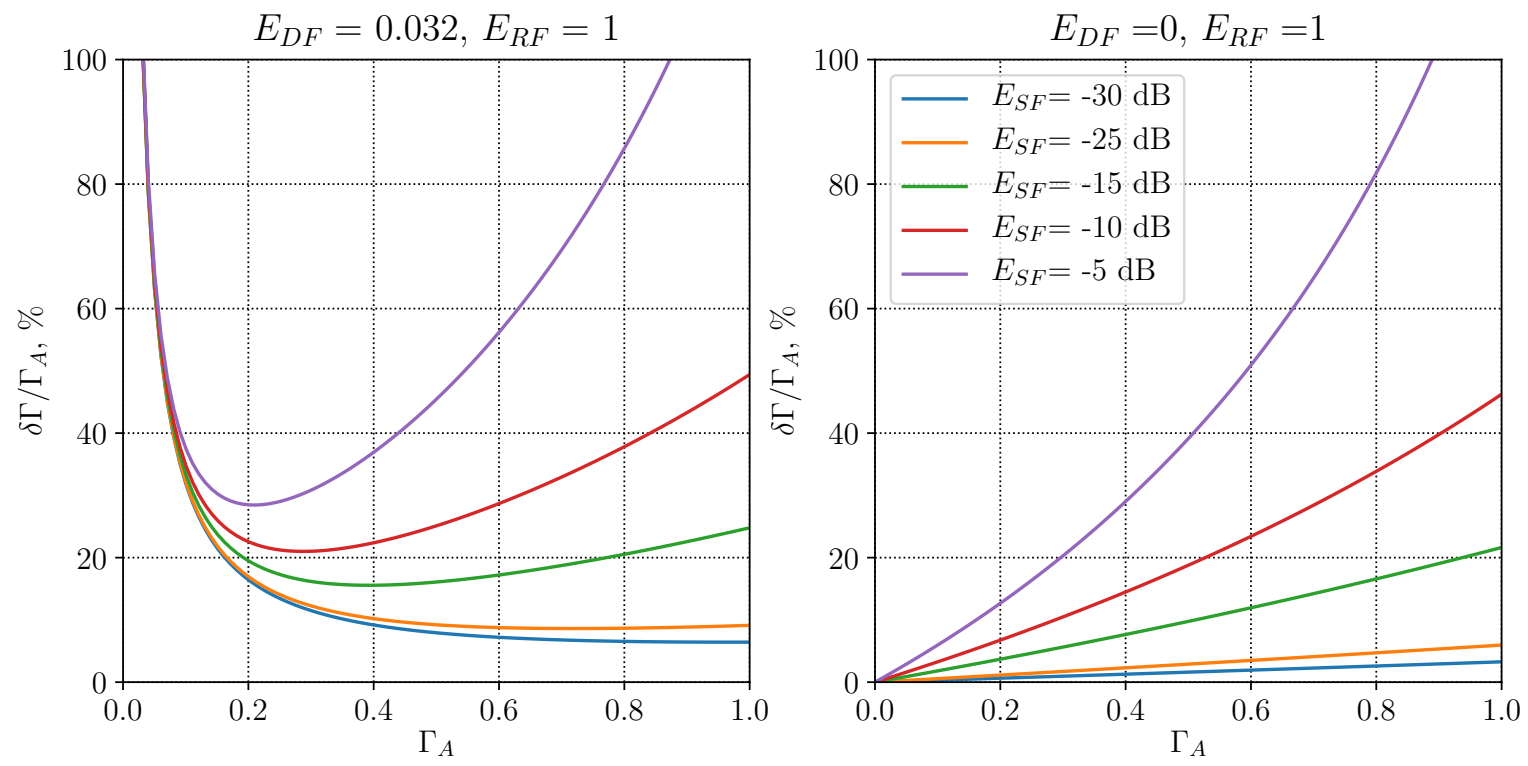

Figure 2: Graphical representation of eq. (6).

Error of reflection measurement with different values of source match $E_{S F}$. Left: with real value of directivity $E_{D F}=0.032$. Right: with ideal directivity $E_{D F}=0$. Both cases assume ideal reflection tracking $E_{R F}=1$.

source match $E_{S F}=-15 \ldots-20 \mathrm{~dB}$, and even for well matched cavity $\Gamma_{A}=0.32$ unexcluded systematic error of cavity input reflection measurement will be around $13 \%$ with calibration procedure from tutorial [1]. 


\subsection{Error in transmission measurement}

Transmission measurement can be defined by model in fig. 3. This model is specific to cavity measurements and is slightly different from general models described in [8] and [9] by fact that cavity transmissions in forward and reverse directions are equal and output port of the cavity has reflection equal to one, since it is very weakly coupled to the cavity. These assumptions are reasonable in almost all practical cases and allow to simplify equations.

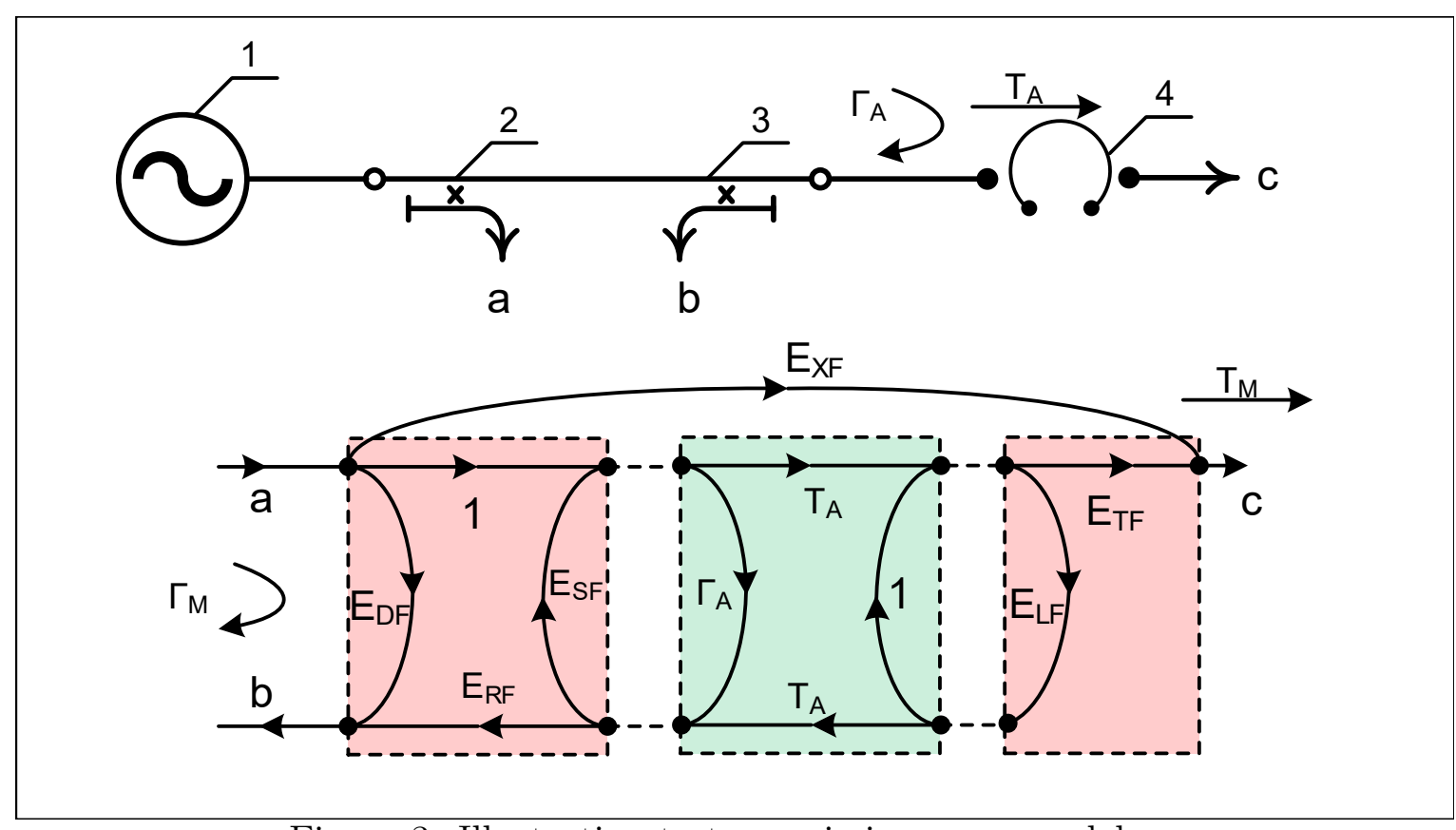

Figure 3: Illustration to transmission error model

1 - r.f. generator; 2 - directional coupler of forward wave; 3 - directional coupler of reflected wave; 4 - cavity under test; $\Gamma_{M}, \Gamma_{A}$ - measured and actual values of reflection from cavity 4 input; $E_{D F}$ - directivity, $E_{R F}$ - reflection tracking, $E_{S F}$ - source match, $T_{M}, T_{A}$ - measured and actual values of cavity 4 trasmission, $E_{L F}$ - load match, $E_{T F}$ - transmission tracking,

$$
E_{X F} \text { - crosstalk. }
$$

From the signal flow graph in fig. 3 the measured value of trasmission $b / a=T_{M}$ can be found as eq. (7) using the non-touching loop rule [6]. By analogy with eq. (6) trasmission error $\delta T$ can be defined as eq. (8).

$$
\begin{gathered}
T_{M}=\frac{T_{A} E_{T F}}{1-\left(\Gamma_{A} E_{S F}+T_{A}^{2} E_{L F} E_{S F}+E_{L F}\right)+\Gamma_{A} E_{S F} E_{L F}}+E_{X F} \\
\delta T=T_{M}-T_{A}
\end{gathered}
$$

Resulting transmission errors are shown in fig. 4, one can see that transmission error is significant and like reflection error it can reach $100 \%$ and even higher in some cases. Calibration procedure [1] allows to exclude only error factor $E_{T F}$ from the measurement, while load match error factor $E_{L F}$ and crosstalk $E_{X F}$ are assumed to be 

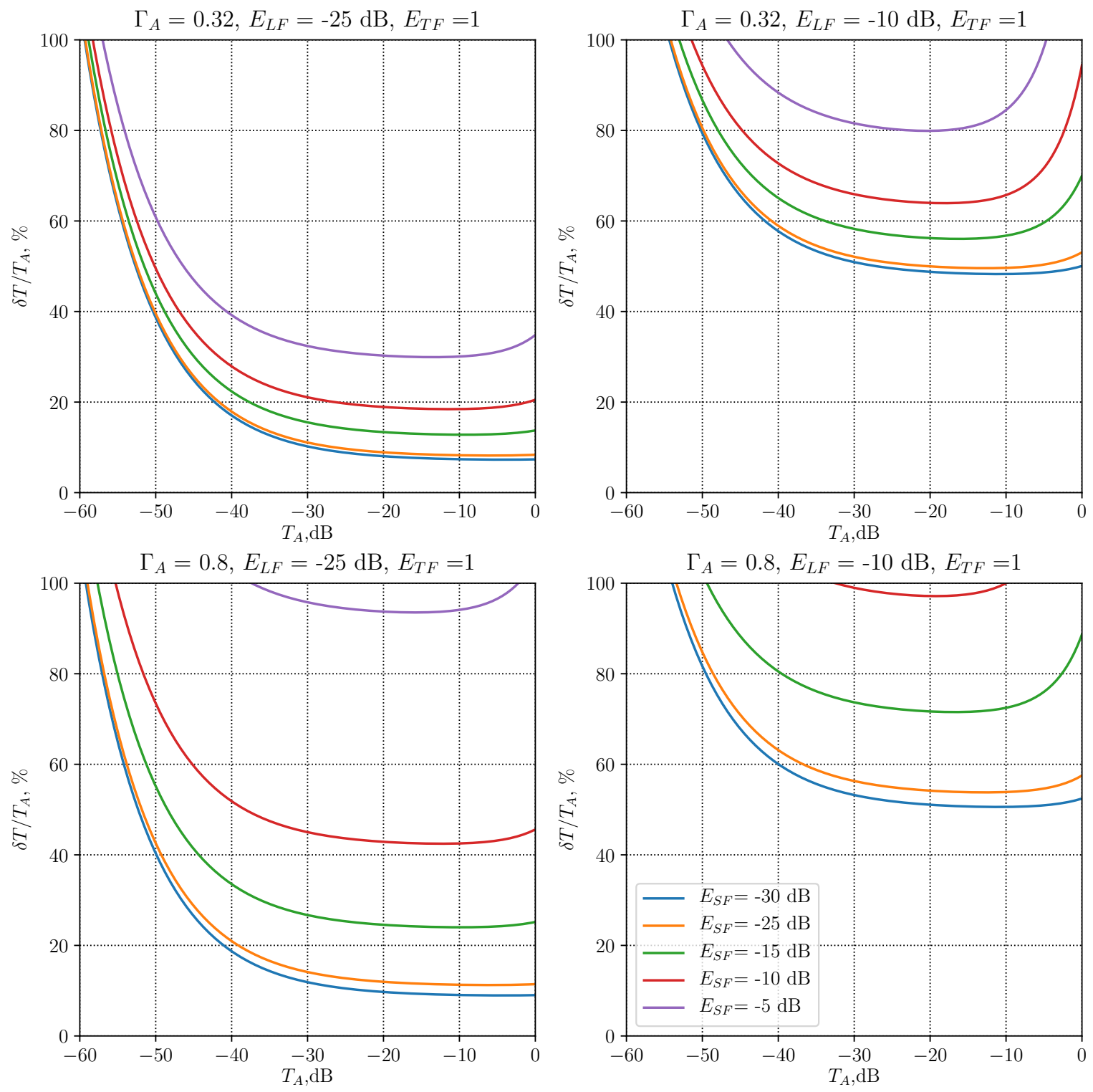

Figure 4: Graphical representation of eq. (8).

Error of transmission measurement with two values of load match $E_{L F}=-30 \mathrm{~dB}$ (excellent load match on the left) and $E_{L F}=-10 \mathrm{~dB}$ (poor load match on the right). And two values of $\Gamma_{A}=0.32$ for relatively good cavity input coupling and $\Gamma_{A}=0.8$ for bad coupling. Different curves correspond to several values of source match $E_{S F}$. Transmission tracking $E_{T F}=1$ is assumed to be known (calibrated). Note that vertical scale is percentage error of the linear value of $T_{A}$, while horizontal scale is logarithmic for convenience.

$$
E_{X F}=-80 \mathrm{~dB} \text { for all curves. }
$$

ideal and equal to zero, however, from blue curve in upper left plot it can be seen, that even in such ideal conditions error is about $5 \%$.

In real operating conditions, as described above when source match $E_{S F}=-15 \ldots-$ $20 \mathrm{~dB}$, cavity input mismatch $\Gamma=0.3 \ldots 0.8$, load match $E_{L F}=-30 \ldots-10 \mathrm{~dB}$ and 
typical cavity attenuation $T_{A}=-20 \mathrm{~dB}$ error will span from $7 \%$ to $60 \%$. Under typical conditions $E_{S F}=-20 \mathrm{~dB}$, load match $E_{L F}=-20 \mathrm{~dB}$ and $\Gamma=0.3 \ldots 0.8$, unexcluded systematic error of cavity transmission measurement will be in range $16 \% \ldots 21 \%$.

These two described above types of errors for transmission and reflection measurements are in essence errors of the coefficients $C_{t}$ and $C_{r}$ in eq. (3). 


\subsection{Errors in $Q_{0}$ measurement}

To evaluate error of $Q_{0}$ measurement we will use eq. (1) and redefine corresponding parameters from eq. (3) and eq. (4). If incident power $P_{i}$ is known (we will show later how to measure it) and parameters $b / a=\Gamma_{M}$ and $c / a=T_{M}$ are measured with setup in fig. 3, then parameters $P_{r}, P_{t}$ and $P_{\text {loss }}$ can be defined in eq. (9). Betas can then be redefined in eq. (10). Note, that absolute values of $\left|\Gamma_{M}\right|$ and $\left|T_{M}\right|$ should be used because $Q_{0}$ is real number.

$$
\begin{aligned}
& P_{r}=P_{i}\left|\Gamma_{M}\right|^{2}, \\
& P_{t}=P_{i}\left|T_{M}\right|^{2}, \\
& P_{\text {loss }}=P_{i}-P_{i}\left|\Gamma_{M}\right|^{2}-P_{i}\left|T_{M}\right|^{2} \\
& \beta_{2}=\frac{\left|T_{M}\right|^{2}}{1-\left|\Gamma_{M}\right|^{2}-\left|T_{M}\right|^{2}}, \\
& \beta^{*}=\frac{1-C_{\beta}\left|\Gamma_{M}\right|}{1+C_{\beta}\left|\Gamma_{M}\right|}, \\
& \beta_{1}=\left(1+\beta_{2}\right) \beta^{*} .
\end{aligned}
$$

By substituting eq. (10) to eq. (1) one will find definition of $Q_{0}$ as eq. (11).

$$
Q_{0_{M}}=\frac{2 Q_{L}\left(C_{\beta}\left|\Gamma_{M}\right|-1\right)}{\left|\Gamma_{M}\right|^{2}+\left|T_{M}\right|^{2}-1}
$$

It is interesting to notice from eq.(11) that $Q_{0}$ measurement in general doesn't rely on the knowledge of absolute power levels, so parameter $P_{i}$ is not needed. To measure $Q_{0_{M}}$ one only needs to know $\Gamma_{M}, T_{M}$ and $Q_{L}$.

Systematic error of $Q_{0_{M}}$ can be calculated as eq. (12).

$$
\delta Q_{0}=\sqrt{\left(\frac{\partial Q_{0_{M}}}{\partial \Gamma_{M}}\right)^{2}|\delta \Gamma|^{2}+\left(\frac{\partial Q_{0_{M}}}{\partial T_{M}}\right)^{2}|\delta T|^{2}+\left(\frac{\partial Q_{0_{M}}}{\partial Q_{L}}\right)^{2} \delta Q_{L}^{2}} .
$$

Corresponding derivatives are:

$$
\begin{aligned}
\frac{\partial Q_{0_{M}}}{\partial \Gamma_{M}} & =\frac{2 Q_{L}\left(\left|T_{M}\right|^{2}-\left|\Gamma_{M}\right|^{2}+2 C_{\beta}\left|\Gamma_{M}\right|-1\right)}{\left(\left|T_{M}\right|^{2}+\left|\Gamma_{M}\right|^{2}-1\right)^{2}} \\
\frac{\partial Q_{0_{M}}}{\partial T_{M}} & =\frac{4 Q_{L}\left|T_{M}\right|\left(1-C_{\beta}\left|\Gamma_{M}\right|\right)}{\left(\left|T_{M}\right|^{2}+\left|\Gamma_{M}\right|^{2}-1\right)^{2}} \\
\frac{\partial Q_{0_{M}}}{\partial Q_{L}} & =\frac{2\left(C_{\beta}\left|\Gamma_{M}\right|-1\right)}{\left|T_{M}\right|^{2}+\left|\Gamma_{M}\right|^{2}-1} .
\end{aligned}
$$

We will further focus on errors $\delta \Gamma$ and $\delta T$ since loaded quality factor mesurement error was discussed in previous works [2]. In this analysis we will consider $Q_{L}$ negligible 
in comparison with $\delta \Gamma$ and $\delta T$, so eq. (12) will reduce to eq. (14):

$$
\delta Q_{0}=\sqrt{\left(\frac{\partial Q_{0_{M}}}{\partial \Gamma_{M}}\right)^{2}|\delta \Gamma|^{2}+\left(\frac{\partial Q_{0_{M}}}{\partial T_{M}}\right)^{2}|\delta T|^{2}} .
$$

An alternative way to estimate errors of $Q_{0}$ measurement is to subtract measured value $Q_{0_{M}}$ from its actual value $Q_{0_{A}}$ by analogy with eqs. (6), (8):

$$
\delta Q_{0}=Q_{0_{M}}-Q_{0_{A}}=\frac{2 Q_{L}\left(C_{\beta}\left|\Gamma_{M}\right|-1\right)}{\left|\Gamma_{M}\right|^{2}+\left|T_{M}\right|^{2}-1}-Q_{0_{A}} .
$$

By substituting $\Gamma_{M}$ and $T_{M}$ in eq. (15) with their definitions from eqs. (5), (7) it is possible to find dependence of $\delta Q_{0}$ on various error factors. Our calculations showed that results obtained from eqs. (15) and (14) differ by less than $3 \%$ in the operating regions (far from singularities). We will use eq. (14) for further investigation.

Analyzing eq. (14) and definitions of $\Gamma_{M}$ and $T_{M}$ one will see that $\delta Q_{0}$ is function of several arguments:

$$
\delta Q_{0}\left(E_{D F}, E_{R F}, E_{S F}, E_{T F}, E_{L F}, E_{X F}, T_{A}, \Gamma_{A}, Q_{L}, C_{\beta}\right)
$$

Let's analyze this result with application to the calibrating procedure from [1]. Error factors $E_{R F}$ and $E_{T F}$ are compensated with it and we assume that standard VTS calibration completely corrects these errors and $E_{R F}=E_{T F}=1$ meaning that corresponding transmission coefficients are normalized. The results of $\delta Q_{0}$ are presented in figure 5. All parameters are in logarithmic scale and have dB units, however in case where we want to show infinite attenuation we write $E_{X X}=0$ in linear units, in the same way we write $E_{X X}=1$ in linear units where we want to show no attenuation.

Plots (a), (c) and (e) show $Q_{0}$ measurement error with standard calibration procedure from [1]. To calculate these curves we assumed directivity of the coupler to be $E_{D F}=-30 \mathrm{~dB}$ and crosstalk $E_{X F}=-60 \mathrm{~dB}$. Plots (a), (c), (e) lead us to an important result. Even with good matching $E_{S F}=E_{L F}=-25 \mathrm{~dB}$ which is hard to achieve in practice, well coupled cavity and all standard scalar calibrations [1] performed, minimum error of $Q_{0}$ measurement is at the order of $10 \%$.

Under conditions closer to real world from series (a) it can be seen that with typical matching of the source and load port at the level of $E_{S F}=E_{L F}=-20 \ldots-15 \mathrm{~dB}$ error of $Q_{0}$ measurement reaches $20 \%$, and is right on the slope of the curve. If $E_{S F}$ changes due to the directional coupler heating etc., total error will increase very fast. Similar result can be seen from series (c). Crosstalk $E_{X F}$, if kept within typical range of $-60 \ldots-50 \mathrm{~dB}$, doesn't change total error much.

These results, however, are true for relatively well matched cavity $\Gamma_{A}=0.464$. If cavity is not coupled well, for example $\Gamma_{A}=0.8$ unexcluded systematic error of $Q_{0}$ measurement will increase $100 \%$, and with real practical parameters of $E_{S F}, E_{L F}$ even several hundred percent.

Plots (b) and (d) show how $Q_{0}$ error can be reduced if calibration procedure could compensate for directivity $E_{D F}$ and crosstalk $E_{X F}$. For the same ideal conditions as above, minimum unexcluded systematic error will reduce from $10 \%$ to almost zero. 
For above real conditions, it reduces from $20 \%$ to about $5 \%$. But if $E_{D F}$ and $E_{X F}$ errors could also be compensated, then total systematic error can be reduced to values below $1 \%$, where random errors will dominate.

This means that if a special calibration procedure based on the above equations is applied, even strongly overcoupled or undercouled cavities can be tested in VTS with relatively small uncertainties . 

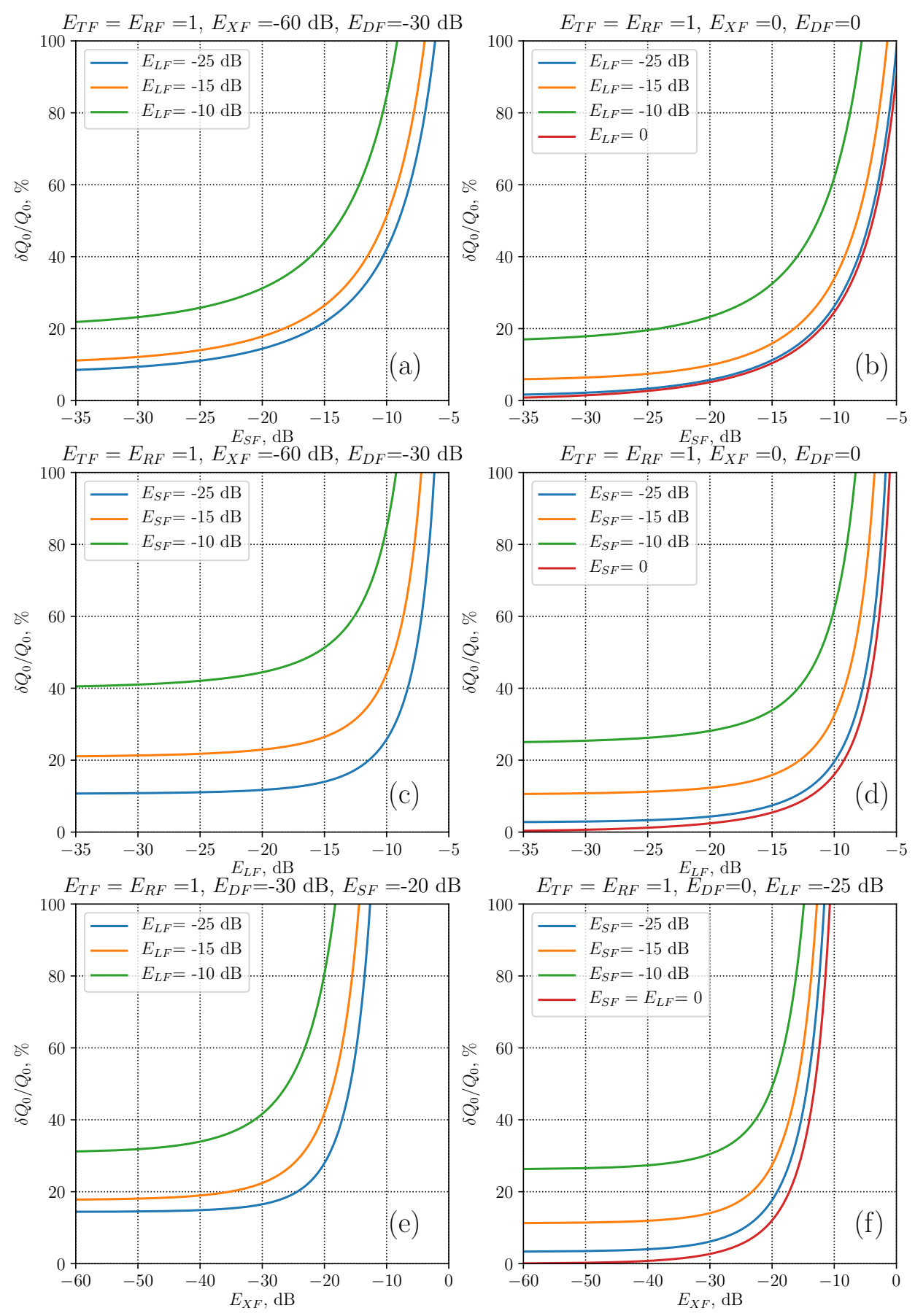

Figure 5: Percentage of $\delta Q_{0}$ depending on $E_{S F}, E_{L F}$ and $E_{X F}$.

(a), (c), (e): only $E_{T F}$ and $E_{R F}$ error factors are compensated (standard VTS calibration); (b), (d), (f): also directivity $E_{D F}$ is compensated. Span $E_{L F}, E_{S F} E_{X F}$ under different conditions. Results are calculated with the next parameters: $Q_{L}=6.162 \cdot 10^{9}, C_{\beta}=-1$, $\Gamma_{A}=0.464, T_{A}=0.26$. Attention: most parameters are in $\mathrm{dB}$, however we use linear units if $E_{X X}=1$ or $E_{X X}=0$. 


\subsection{Errors in the gradient measurement}

Using gradient eq. (2) by analogy with (12) we will define error of gradient measurement as eq. (17), assuming that uncertainty of coefficient $\kappa=\sqrt{\frac{r / Q}{L}}$ is negligibly small in comparison with other uncertainties, as it was found in [4].

$$
\begin{aligned}
& \delta E_{\text {acc }}=\sqrt{\left(\frac{\partial E_{a c c}}{\partial Q_{0}}\right)^{2} \delta Q_{0}^{2}+\left(\frac{\partial E_{a c c}}{\partial P_{l o s s}}\right)^{2} \delta P_{\text {loss }}^{2}}= \\
& =\sqrt{\left(\frac{P_{\text {loss }} \kappa}{2 \sqrt{Q_{0} P_{\text {loss }}}}\right)^{2} \delta Q_{0}^{2}+\left(\frac{Q_{0} \kappa}{2 \sqrt{Q_{0} P_{\text {loss }}}}\right)^{2} \delta P_{\text {loss }}^{2}}= \\
& =\frac{1}{2} \sqrt{\frac{\kappa^{2}\left(\delta Q_{0}^{2} P_{\text {loss }}^{2}+\delta P_{\text {loss }}^{2} Q_{0}^{2}\right)}{P_{\text {loss }} Q_{0}}}
\end{aligned}
$$

As can be seen from eq. (17) it demands for uncertainty $\delta P_{\text {loss }}$. This uncertainty can be found by differentiating equation for $P_{\text {loss }}$ in (9):

$$
\begin{aligned}
& \frac{\partial P_{\text {loss }}}{\partial P_{i}}=1-\left|T_{M}\right|^{2}-\left|\Gamma_{M}\right|^{2} \\
& \frac{\partial P_{\text {loss }}}{\partial\left|T_{M}\right|}=-2 P_{i}\left|T_{M}\right| \\
& \frac{\partial P_{\text {loss }}}{\partial\left|\Gamma_{M}\right|}=-2 P_{i}\left|\Gamma_{M}\right|
\end{aligned}
$$

Then using (18), uncertainty $\delta P_{\text {loss }}$ can be found as eq.(19) by analogy with $\delta E_{a c c}$ :

$$
\begin{aligned}
& \delta P_{\text {loss }}=\sqrt{\left(\frac{\partial P_{\text {loss }}}{\partial P_{i}}\right)^{2} \delta P_{i}^{2}+\left(\frac{\partial P_{\text {loss }}}{\partial\left|T_{M}\right|}\right)^{2}|\delta T|^{2}+\left(\frac{\partial P_{\text {loss }}}{\partial\left|\Gamma_{M}\right|}\right)^{2}|\delta \Gamma|^{2}}= \\
& =\sqrt{\delta P_{i}^{2}\left(\left|T_{M}\right|^{2}+\left|\Gamma_{M}\right|^{2}-1\right)^{2}+4 P_{i}^{2}\left|T_{M}\right|^{2}|\delta T|^{2}+4 P_{i}^{2}\left|\Gamma_{M}\right|^{2}|\delta \Gamma|^{2}}
\end{aligned}
$$

Analyzing eqs. $(19,17)$ and definitions of $\Gamma_{M}$ and $T_{M}$ one will see that $\delta E_{a c c}$ is function of several arguments:

$$
\delta E_{a c c}\left(E_{D F}, E_{R F}, E_{S F}, E_{T F}, E_{L F}, E_{X F}, T_{A}, \Gamma_{A}, Q_{L}, C_{\beta}, P_{i}, \delta P_{i}, \kappa\right) .
$$

The results of fractional uncertainty eq. (17) under various conditions are given in fig. 6. All parameters are in logarithmic scale and have dB units, however in case where we want to show infinite attenuation we write $E_{X X}=0$ in linear units, in the same way we write $E_{X X}=1$ in linear units where we want to show no attenuation.

These results in fig. 5 and in fig. 6 were calculated for cavity TE1PAV007 tested in VTS on October 15, 2019 for one point, where measured parameters were $E_{a c c}=10.51$ $\mathrm{MV} / \mathrm{m}, Q_{0}=2.516 \cdot 10^{10}$. Lets analyze fig. 6. Series (a), (c), (e) show uncertainty with 
standard VTS calibration procedure from [1] and various values $E_{S F}, E_{L F}$ and $E_{X F}$. It can be seen from (a) that even under approaching to ideal conditions when source match $E_{S F}=-30 \mathrm{~dB}$ gradient uncertainty is in range of $5 \%$. Under real conditions when $E_{S F}=-20 \ldots-15 \mathrm{~dB}$, gradient error is in range of $10 \%$ if load match $E_{L F}$ is good, and error can reach $20 \%$ if match $E_{L F}$ is poor. Series (b) show that if directivity $E_{D F}$ and cross-talk $E_{X F}$ corrections are applied then corresponding uncertainties reduce from $10 \%$ to $4 \%$ for good match $E_{L F}$ and from $20 \%$ to $17 \%$ for poor match $E_{L F}$.

Then, red trace in series (b) at $-40 \mathrm{~dB}$ point shows the condition when all corrections are applied and total uncertainty reduces to $2.5 \%$, when only uncertainty $\delta P_{i}$ remains. Finally, if we set $\delta P_{i}=0$ (which cannot be done in reality) total uncertainty will also reduce to $0 \%$ (red dotted line), which shows that model works correctly.

Series (c), (d), (e), (f) show that uncertainty curves are relatively flat in the operating ranges of parameters $E_{L F}=-25 \ldots-15 \mathrm{~dB}$, and $E_{X F}=-60 \ldots-40 \mathrm{~dB}$. However, in the operating range of source match $E_{S F}=-20 \ldots-15 \mathrm{~dB}$ ratio $\delta E_{a c c} / E_{\text {acc }}$ becomes very non-linear. This means that source match significantly effects the error of gradient measurement. In general, series (a) show that without additional correction VTS calibration [1] can lead up to $15 \%$ error in gradient measurement, even for relatively well matched cavity. For strongly over-coupled or under-coupled cavity this error will be sufficiently higher. 

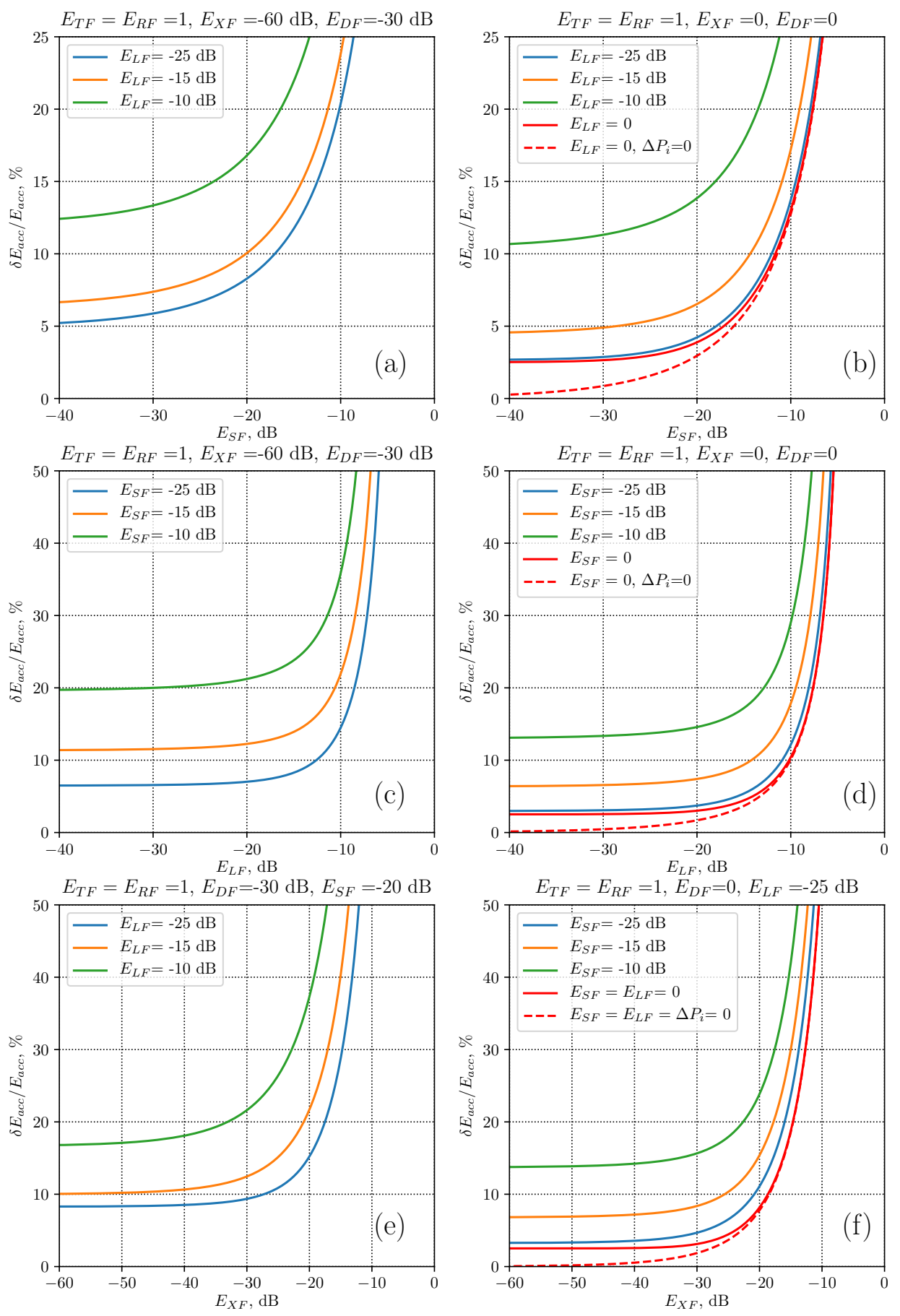

Figure 6: Percentage of $\delta E_{a c c}$ depending on $E_{S F}, E_{L F}$ and $E_{X F}$.

(a), (c), (e): only $E_{T F}$ and $E_{R F}$ error factors are compensated (standard VTS calibration); (b), (d), (f): also directivity $E_{D F}$ is compensated. Results are calculated with the next parameters: $Q_{L}=6.162 \cdot 10^{9}, C_{\beta}=-1, \Gamma_{A}=0.464, T_{A}=0.26, \kappa=88.474, P_{i}=0.76 \mathrm{~W}$, $\delta P_{i}=5 \%$. Attention: most parameters are in $\mathrm{dB}$, however we use linear units if $E_{X X}=1$ or $E_{X X}=0$. 


\subsection{Vector errors}

Let's now analyze what effect deviations in cable length have on errors of $Q_{0}$ and $E_{a c c}$ measurement. In general, equations (5) and (7) demand for complex values of $E_{D F}, E_{R F}, E_{S F}, E_{T F}, E_{L F}, E_{X F}$, and corresponding values of $\Gamma$ and $T$ and their errors $\delta \Gamma$ and $\delta T$ are complex by definition. This is why all formulas for $Q_{0}, E_{a c c}$, $\delta Q_{0}, \delta E_{a c c}$ contain only absolute values of $\Gamma, T, \delta \Gamma$ and $\delta T$. However, error factors $E_{D F}, E_{R F}, E_{S F}, E_{T F}, E_{L F}, E_{X F}$ add and multiply as vectors and therefore absolute values of $\Gamma, T, \delta \Gamma$ and $\delta T$ and as a result values of $\delta Q_{0}, \delta E_{a c c}$ are functions not only of magnitudes, but also phases (arguments). This means that errors of $Q_{0}$ and $E_{a c c}$ directly depend on variations in electrical lengths of the components in VTS. In [2] authors used heuristic approach to show this dependency for $E_{R F}$ error factor. By using complex error factors in equations from above sections of this work, one can get strict and complete analytic definitions of VTS vector errors for any of $E_{D F}, E_{R F}, E_{S F}, E_{T F}, E_{L F}, E_{X F}$ parameters. We will show it next.

For our example cavity TE1PAV007 resonance frequency is $f=1.3 \mathrm{GHz}$. Any error factor $E_{X X}$ can be defined using complex notation (21).

$$
E_{X X}(A, L)=A \cdot e^{j \beta L}
$$

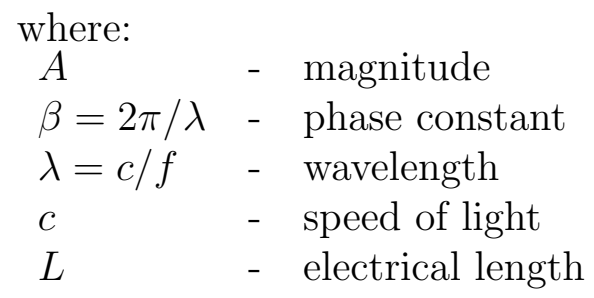

Previously we found, that standard VTS calibration procedure [1] corrects for $E_{R F}$ and $E_{T F}$ errors, but what if electrical length of the r.f. connections change due to cable bending or temperature variations, or bad r.f. connectors, or even if one add a small r.f. adapter between directional coupler and test cable? Procedure [1] will not calibrate such errors.

Figure 7 shows what happens. From series (a) and (c) one can find that for 1.3 $\mathrm{GHz}$ cavity, change in electrical length of the cable from zero to $100 \mathrm{~mm}$ will lead to change in uncertainty from $8 \%$ to $100 \%$ in gradient and from $14 \%$ to $180 \%$ in quality factor. Effect from electrical length variations in $E_{S F}, E_{L F}$, and $E_{D F}$ is not so catastrophic, but also leads to uncertainty variations about $8 \%$. Note the behavior of the curves: they oscillate because standing wave is shifting in transmission line together with increase of the distance between generator and load - very well known effect.

Of course, in normal conditions length of the cables should not change after calibrations so much. But with increase in cavity frequency this oscillating region will shift toward left and even very small variations in length will cause significant variations in uncertainty.

However, even for $1.3 \mathrm{GHz}$ what seems a small modification in hardware can have significant impact. Assume one adds a N-male-to-N-male adapter to the cavity $P_{i}$ cable after calibration was performed to connect it back to the directional coupler. Such adapter has length about $10 \mathrm{~mm}$, this will change electrical length of $E_{R F}$ error 
factor. As can be seen from figure 8, such change in hardware will cause uncertainty of $Q_{0}$ to increase from $15 \%$ to $35 \%$, and $E_{a c c}$ from $8 \%$ to $20 \%$.
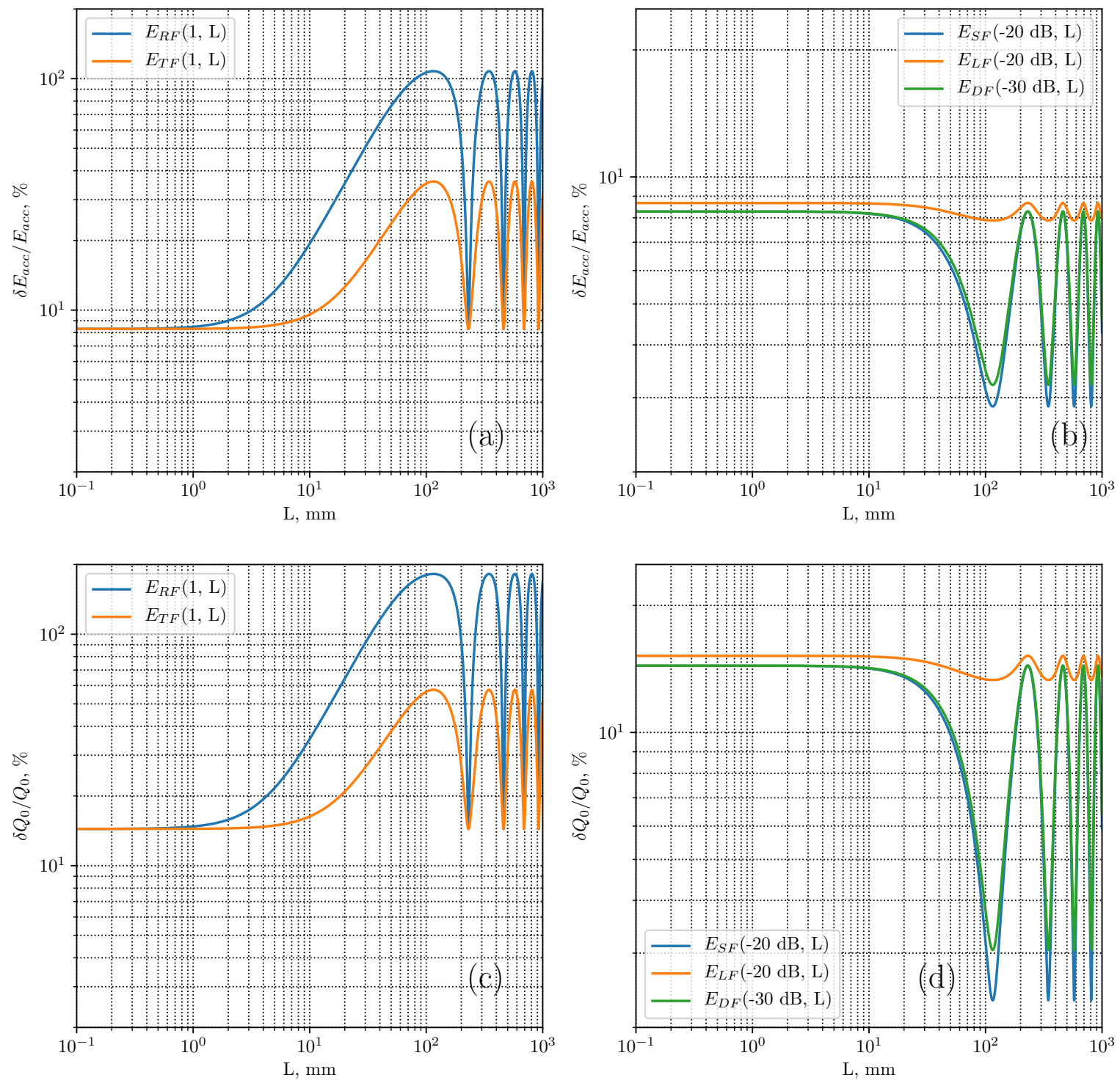

Figure 7: Fractional uncertainties of gradient $E_{a c c}$ and $Q_{0}$ measurement depending on electrical length $L$ of various error factors.

(a), (b): fractional uncertainty of gradient measurement, (c), (d): fractional uncertainty of $Q_{0}$ measurement. For all curves standard VTS calibration [1] applied, with no vector error correction. Data calculated for $1.3 \mathrm{GHz}$ over-coupled cavity, $\Gamma_{A}=0.464$ (TE1PAV007). 

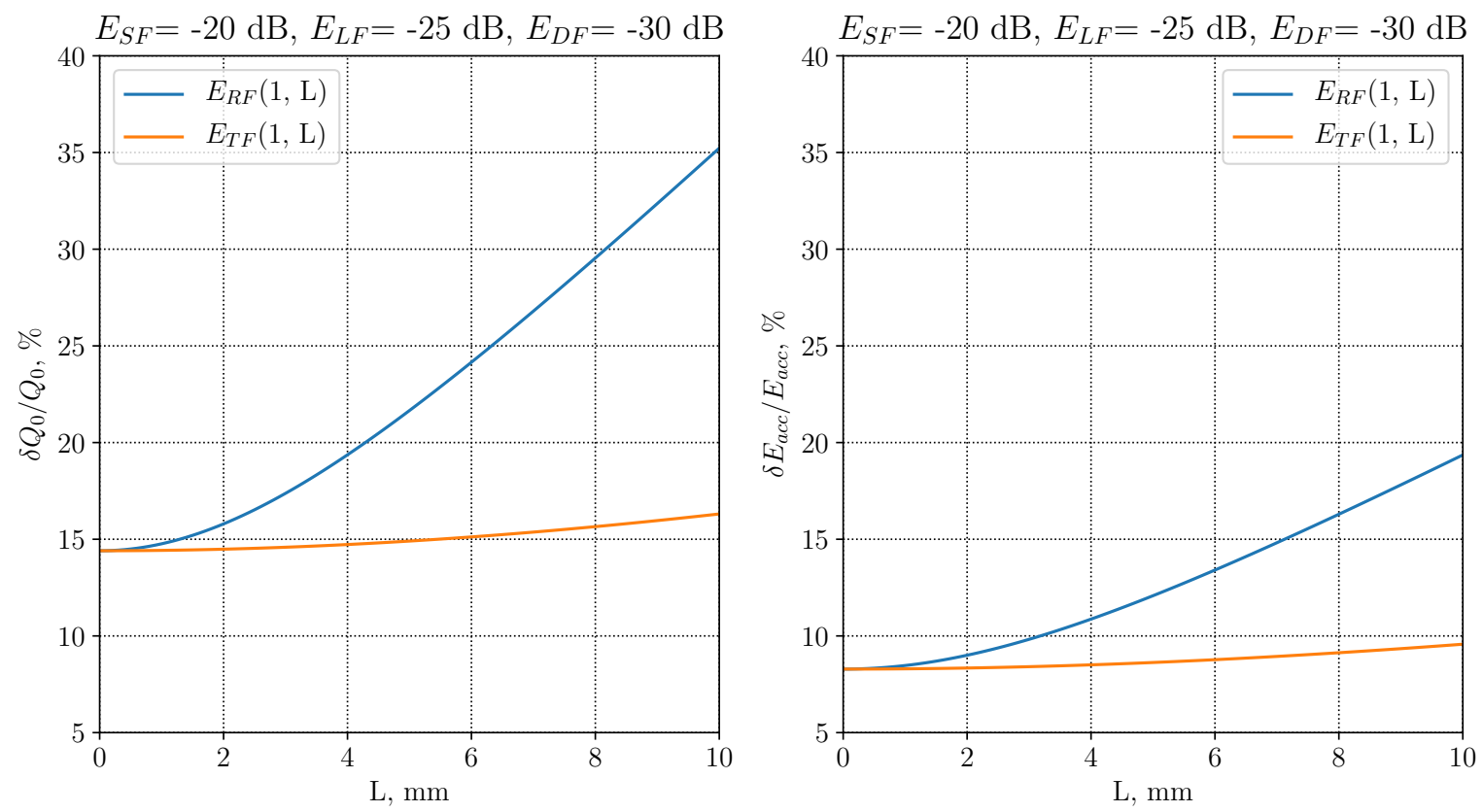

Figure 8: Fractional uncertainties of gradient $E_{a c c}$ and $Q_{0}$ measurement depending on electrical length $L$ of $E_{R F}$ and $E_{T F}$.

Zoomed versions of (a) and (c) plots of fig. 7. With standard VTS calibration [1], no vector error correction. Data calculated for $1.3 \mathrm{GHz}$ over-coupled cavity, $\Gamma_{A}=0.464$ (TE1PAV007). 


\subsection{Summary on errors}

Using error models for transmission and reflection measurement and graph theory we derived complete analytical relationships between error terms and measured values of $Q_{0}$ and $E_{a c c}$ that show how actual values will change due to impact of errors. We demonstrated that systematic errors of $Q_{0}$ and $E_{\text {acc }}$ measurement can be characterized by six error terms: $E_{D F}, E_{R F}, E_{S F}, E_{T F}, E_{L F}, E_{X F}$. Standard VTS calibration procedure corrects only for two of these terms: $E_{R F}, E_{T F}$ and provides only scalar correction.

While for critically matched cavity this can be enough, in real world conditions ignoring of other terms will cause systematic error that can span form $15 \%$ to several hundreds in $Q_{0}$ and from $8 \%$ to several tens for $E_{a c c}$. For example we used VTS raw data of real cavity and processed it with our equations, applying practical values of remaining error terms: $E_{D F}, E_{S F}, E_{L F}, E_{X F}$, assuming that $E_{R F}=E_{T F}=1$ (calibrated).

The resulting $Q_{0}$ plot with error bars is given in fig. 9. To get this plot we took $Q_{0}\left(E_{a c c}\right)$ data from VTS, assuming it to be perfect "actual data". Then we added errors to this data based on our analytic model and real hardware parameters of the test stand resulting in distorted data "measured data". However, result in fig. 9 is only simulation; in reality, VTS raw data already has errors and real hardware experiments should be conducted to show how scalar corrected data will differ from vector corrected data.

From fig. 9 one can see that reduction of reflection from the cavity input $\Gamma$ leads to reduction in total error of $Q_{0}$ and $E_{a c c}$, as expected from previous works [1], [2]. In this plot error of power meter is assumed $\delta P_{i}=5 \%$, directivity $E_{D F}=-30 \mathrm{~dB}$, source match $E_{S F}=-20 \mathrm{~dB}$, load match $E_{L F}=-25 \mathrm{~dB}$. Depending whether cavity is under-coupled or over-coupled this errors will create positive or negative bias to the final $Q_{0}$ result.

These systematic errors can be measured and corrected, reducing the total error of measurement to negligible random errors. Methods and mathematics of such corrections and required hardware modifications to VTS are described in the next sections. 

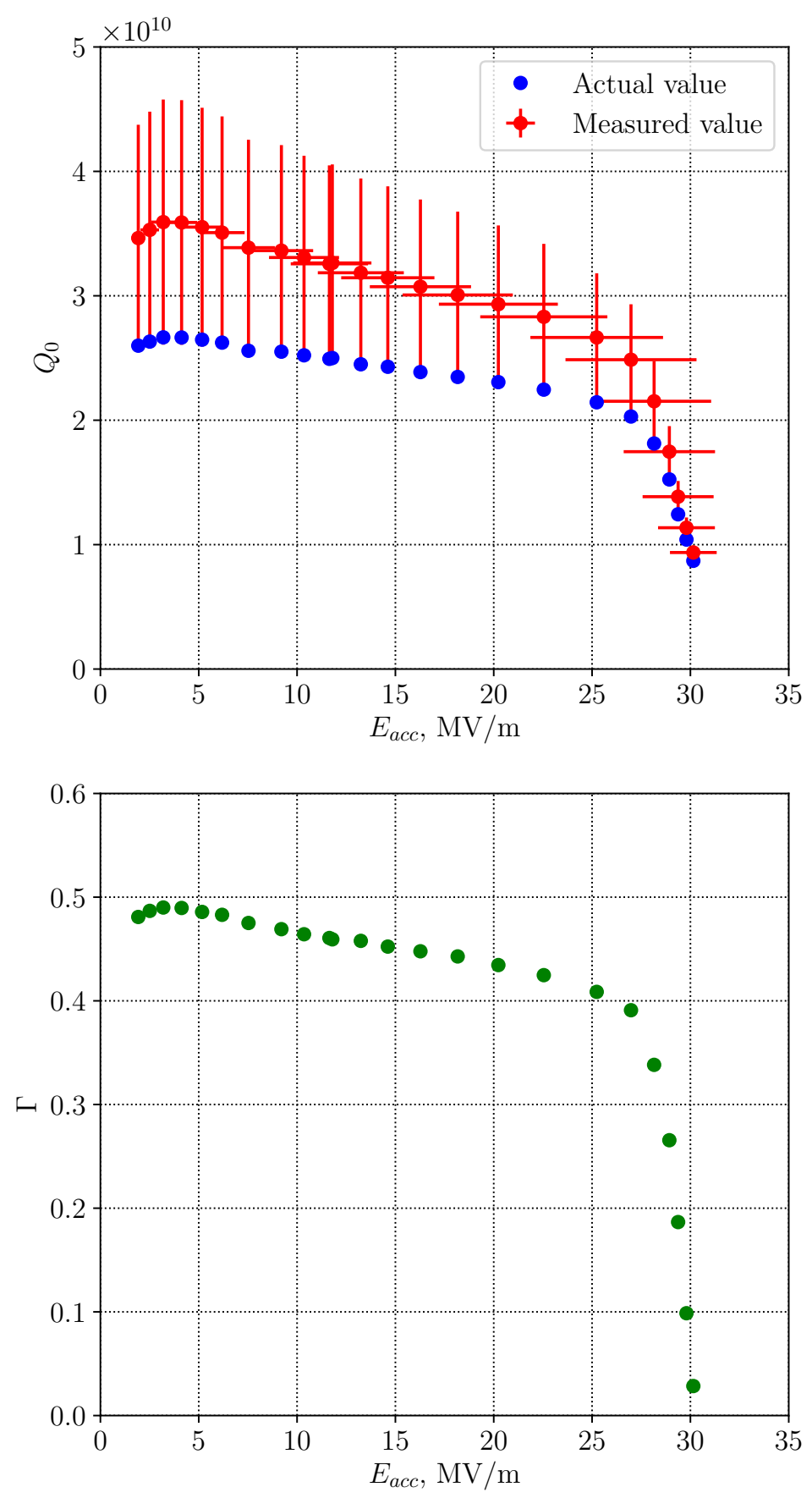

Figure 9: Top: cavity parameters as measured at VTS (blue) and with added erros as calculated (red); bottom: measured reflection from cavity input. 


\section{Improved Vertical Test Stand Architecture}

A block diagram of the vertical test stand r.f. system that allows to perform a vector error correction is shown in fig. 10 .

The r.f. system works as follows. In the normal cavity measurement mode switch 13 is in position "4" and matrix switch 19 is in position "3-2". In such configuration generator 1 produces an r.f. signal that goes through transmitter " $T$ " where it is amplified in the power amplifier 4, and then through switch 13 and test cable 17, and coupler 21 it finally reaches cavity 22. Some of the power is reflected back from the cavity 22 and coupler 21 and returns back to the transmitter "T" through test cable 17 and switch 13, where it is directed to downconverter 9 through attenuator 8 and circulator 7 . Some portion of r.f. power from the output of the amplifier 4 is also directed to the downconverter 6 through directional coupler 5. Power from the coupler 23 is directed to the downconverter 11 trough the test cable 20, switch 19 and broadband splitter 12, the rest part of this power goes through splitter 12 to the phase locked loop 3. This phase locked loop generates control signal that is proportional to the cavity 22 detuning from the frequency of the r.f. generator 1 . Control signal tunes generator 1 in resonance with cavity 22, so a stable magnitude of electric field can be achieved in the cavity, like in any known SRF test system.

An additional r.f. generator 2 has frequency offset by several $\mathrm{MHz}$ from generator 1 . Generator 2 serves as a local oscillator for downconverters 6, 9, 10. Generators 1 and 2 can be locked so frequency offset between them always remains the same, which means that downconverters will produce signals with equal frequencies on their outputs. This signals can be then digitized and processed in digitizer 10 in order to find their vector ratios.

In the calibration mode parameters of the transmitter " $\mathrm{T}$ " are measured by comparing magnitude and phase of signals from the outputs of downconverters 6,9 with different standard loads connected, by turning switch 13 into corresponding positions. Parameters of test cable 17 are also measured during this step.

Then switch 13 is turned into position 5, and switch 19 into position "1-2" parameters of test cable 20 are measured in the same way by comparing signals from the outputs of the downconverters 6,9 .

Finally, switch 19 is turned into position "1-3" to measure parameters of the receiver "R" by comparing magnitude and phase of signals from the outputs of downconverters 6, 11. This finalizes calibration procedure, from now switch 19 is turned into position "3-2" to do regular cavity measurements. In the following sections we will describe this calibration procedure in details. 


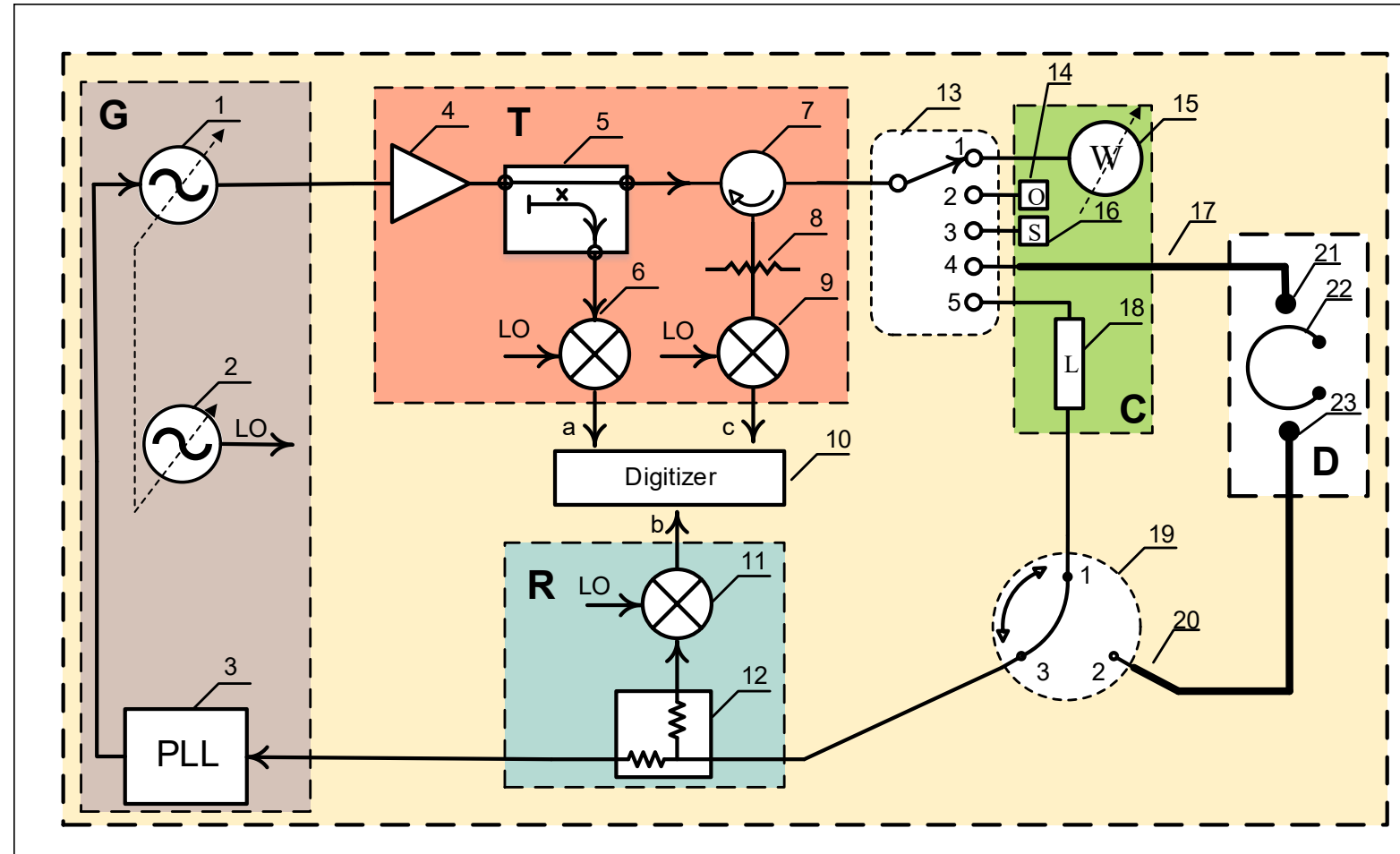

Figure 10: Block-diagram of improved VTS system

1,2 - r.f. generators; 3 - phase-locked-loop; 4 - r.f. power amplifier; 5 - directional coupler;

7 - high power circulator; 8 - attenuator; 6,9,11 - downconverters; 10 - digitizer;

12 - broadband splitter; 13 - SP5T r.f. switch; 15 - r.f. power meter; 14 - open standard;

16 - short standard; 18 - transmission standard; 17,20 - test cables (including cryogenic

part), 19 - three-port matrix switch; 21 - input power coupler; 22 - cavity; 23 - transmitted power coupler; "T" - transmitter; "R" - receiver; "G" - signal generation section;

"C" - calibration standards' section; "D" - dewar.

\section{$5 \quad$ VTS r.f. error model and de-embedding}

Flow graph in fig. 11 shows a complete VTS error model. When VTS is operated in normal mode the electronics is measuring voltages in three points $a, b$ and $c$. In fig. 10 these points are outputs of the downconverters 6,11 and 9 correspondingly. If there were no error factors in the system direct ratios of $b / a$ and $c / a$ would give one the trasmission through the cavity and reflection from the cavity.

However under real conditions there are error terms, that exist between cavity and the meters that distort measurement result. These error terms are $E_{D F}, E_{R F}, E_{S F}$ of the transmitter "T", $E_{T F}, E_{L F}$ of receiver "R", $E_{X F}$ - crosstalk, $T_{I}, \Gamma_{I W}, \Gamma_{I C}$ of the cable 17 and $T_{T}, \Gamma_{T C}, \Gamma_{T W}$ of the cable 20 . It is necessary to find these paprameters in order to exclude them from model and finally extract parameters $T$ and $\Gamma$ of the cavity.

Unfortunately, there are several practical problems since parameters $\Gamma_{I W}, \Gamma_{I C}$, $\Gamma_{T W}, \Gamma_{T C}$ cannot be measured directly without placing calibration standards in cryogenic environment like authors did it in [10], mainly because it is not studied how 


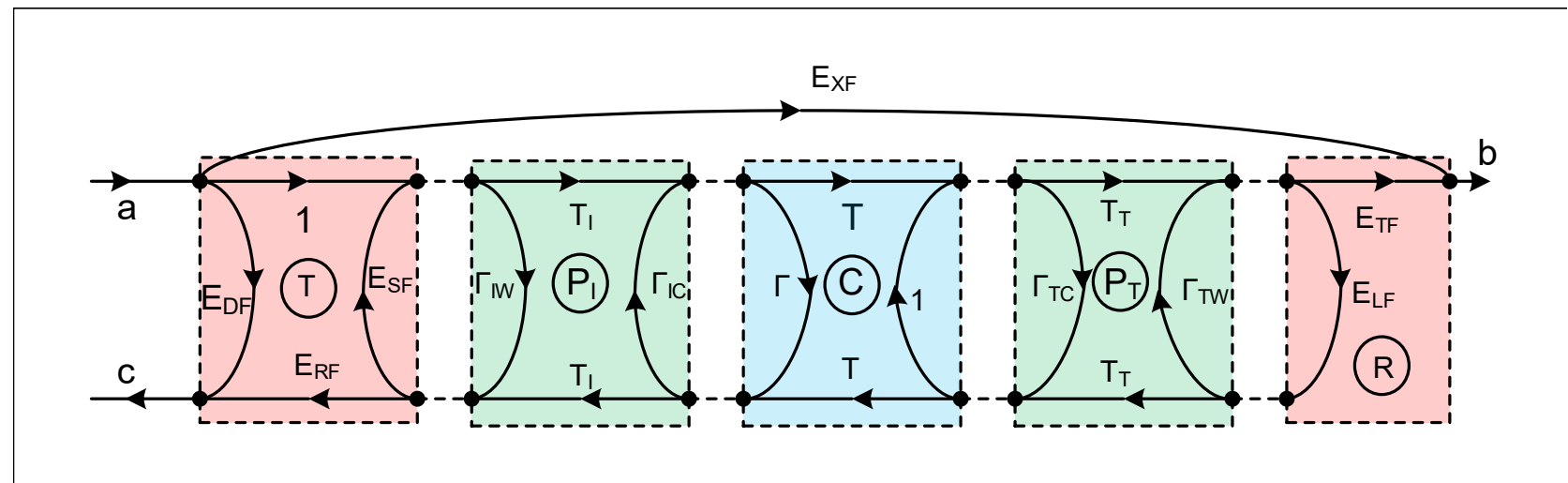

Figure 11: Flow graph of cavity measurement in system of S-parameters

$\begin{array}{lll}T, R & - & \text { transmitter and receiver } \\ P_{I}, P_{T}, C & - & \text { incident, transmitted power cables and cavity } \\ & & \text { with corresponding S-parameters } \\ E_{D F} & - & \text { directivity } \\ E_{R F} & - & \text { reflection tracking } \\ E_{S F} & - & \text { source match } \\ E_{T F} & - & \text { transmission tracking } \\ E_{L F} & - & \text { load match } \\ E_{X F} & - & \text { crosstalk }\end{array}$

high power r.f. switches will operate in liquid helium and what will be the operation reliability.

However from series (d) in fig. 6 and fig. 5 it can be seen that if $E_{L F}$ is in range $-30 \ldots-20 \mathrm{~dB}$ it doesn't change the value of total error, therefore it can be ignored, and it is practically possible to make $E_{L F}$ be close to zero. Then $\Gamma_{T W}, \Gamma_{T C}$ can also be ignored. Finally, if cavity reflection $\Gamma>\Gamma_{I W}, \Gamma_{I C}$ which is true in most practical scenarios then $\Gamma_{I W}$ and $\Gamma_{I C}$ can also be ignored since they will make very small impact to the error of $Q_{0}$ and $E_{a c c}$ measurement. Such assumptions are rather reasonable since cable connectors can be made with very high quality, and good load match $E_{L F}$ of the receiver " $\mathrm{R}$ " is achieved with high quality splitter 12 and switch 19 . This allows to significantly simplify flow graph from fig.11. This reduced graph is shown in fig. 12

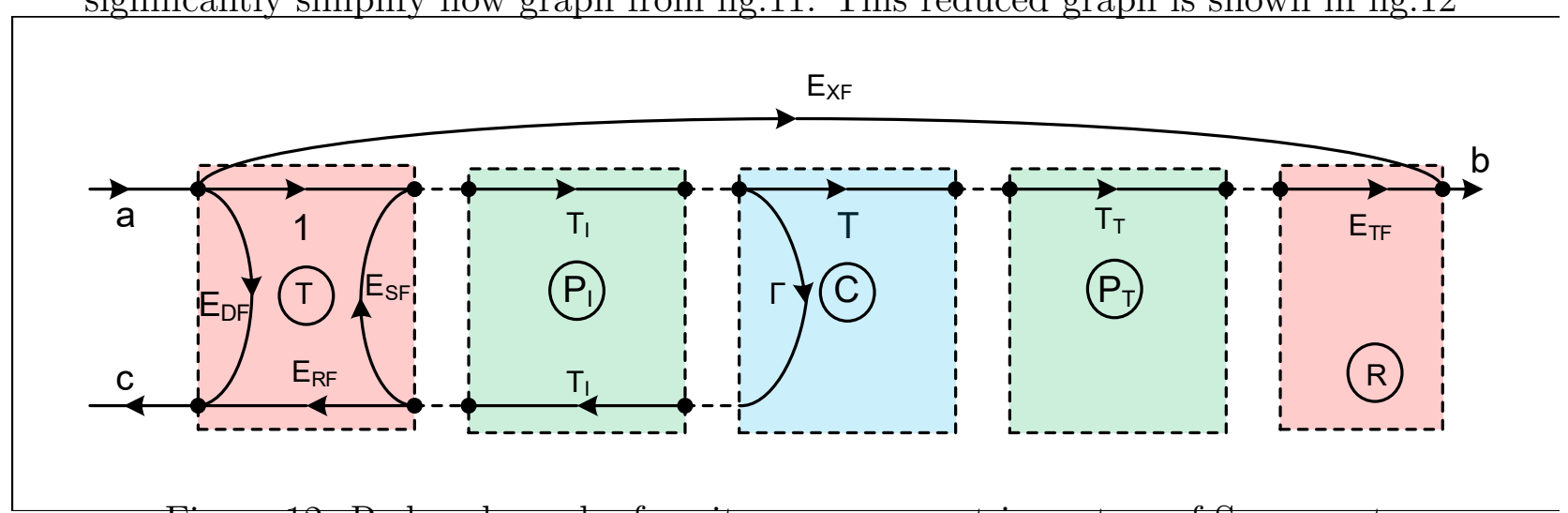

Figure 12: Reduced graph of cavity measurement in system of S-parameters

From this graph measured reflection $c / a=M_{\Gamma}$ from the cavity can be defined as eq. (22). 


$$
M_{\Gamma}=E_{D F}+\frac{T_{I}^{2} \Gamma E_{R F}}{1-\Gamma T_{I}^{2} E_{S F}}
$$

And transmission $b / a=M_{T}$ through the cavity can be defined as eq.(23).

$$
M_{T}=E_{X F}+\frac{T_{I} T T_{T} E_{T F}}{1-\Gamma T_{I}^{2} E_{S F}}
$$

By solving system of eqs. $(22,23)$ one can easily find final cavity parameters:

$$
\begin{gathered}
\Gamma=\frac{M_{\Gamma}-E_{D F}}{E_{R F} T_{I}^{2}-E_{D F} E_{S F} T_{I}^{2}+E_{S F} T_{I}^{2} M_{\Gamma}} \\
T=\frac{E_{R F} M_{T}-E_{R F} E_{X F}}{E_{R F} E_{T F} T_{I} T_{T}-E_{D F} E_{S F} E_{T F} T_{I} T_{T}+E_{S F} E_{T F} T_{I} T_{T} M_{\Gamma}}
\end{gathered}
$$

\section{VTS r.f. system vector calibration}

Eqs. $(24,25)$ show that transmitter parameters $E_{S F}, E_{D F}$ and $E_{R F}$; receiver parameter $E_{T F}$; and cable parameters $T_{I}, T_{T}$ must be known. These parameters can be measured using OSM (Open Short Match, sometimes reffered also as SOL) calibration [11], [9], [8]. Before calibration, actual S-parameters of calibration standards 14,16,18 and reflection coefficient of r.f. power meter 15 should be measured with calibrated vector network analyzer and recorded in files. After that VTS system can be characterized.

\subsection{Transmitter calibration}

First, parameters of transmitter "T" will be found. In calibration section "C" r.f. power meter 15 serves as matched load calibration standard. Switch 13 is turned into position "1" and vector voltage ratio $c / a$ between outputs of mixers 9 and 6 gives measured reflection value of matched standard.

$$
L_{M}=E_{D F}+\frac{E_{R F} L_{A}}{1-E_{S F} L_{A}}
$$

Then switch 13 is turned in position "2" and open standard is measured:

$$
O_{M}=E_{D F}+\frac{E_{R F} O_{A}}{1-E_{S F} O_{A}}
$$

Then switch 13 is turned in position "3" and short standard is measured:

$$
S_{M}=E_{D F}+\frac{E_{R F} S_{A}}{1-E_{S F} S_{A}}
$$

Then solution of system $(26,27,28)$ gives error terms $E_{D F}, E_{R F}, E_{S F}$. 


$$
\begin{aligned}
& N=L_{A} L_{M} O_{A} S_{M}-L_{A} L_{M} O_{M} S_{A}-L_{A} O_{A} O_{M} S_{M}+L_{A} O_{M} S_{A} S_{M}+ \\
& +L_{M} O_{A} O_{M} S_{A}-L_{M} O_{A} S_{A} S_{M} \\
& E_{D F}=\frac{N}{L_{A} L_{M} O_{A}-L_{A} L_{M} S_{A}-L_{A} O_{A} O_{M}+L_{A} S_{A} S_{M}+O_{A} O_{M} S_{A}-O_{A} S_{A} S_{M}} \\
& E_{R F}=\frac{\left(L_{A}-O_{A}\right)\left(L_{A}-S_{A}\right)\left(L_{M}-O_{M}\right)\left(L_{M}-S_{M}\right)\left(O_{A}-S_{A}\right)\left(O_{M}-S_{M}\right)}{\left(L_{A} L_{M} O_{A}-L_{A} L_{M} S_{A}-L_{A} O_{A} O_{M}+L_{A} S_{A} S_{M}+O_{A} O_{M} S_{A}-O_{A} S_{A} S_{M}\right)^{2}} \\
& E_{S F}=\frac{-L_{A} O_{M}+L_{A} S_{M}+L_{M} O_{A}-L_{M} S_{A}-O_{A} S_{M}+O_{M} S_{A}}{L_{A} L_{M} O_{A}-L_{A} L_{M} S_{A}-L_{A} O_{A} O_{M}+L_{A} S_{A} S_{M}+O_{A} O_{M} S_{A}-O_{A} S_{A} S_{M}}
\end{aligned}
$$

\subsection{Crosstalk calibration}

Next, switch 13 is turned into position "1" and switch 19 into position "3-2" and measurement of vector voltage ratio $b / a$ between outputs of mixers 11 and 6 gives crosstalk $E_{X F}$ directly.

\subsection{Receiver calibration}

Next, parameters of receiver " $\mathrm{R}$ " are found: switch 13 is turned to position " 5 " and switch 19 to posotion "1-3". A transmission standard 18 with known S-parameters is now connected between transmitter "T" and receiver "R". Flow graph of such connection is shown in fig. 13 .

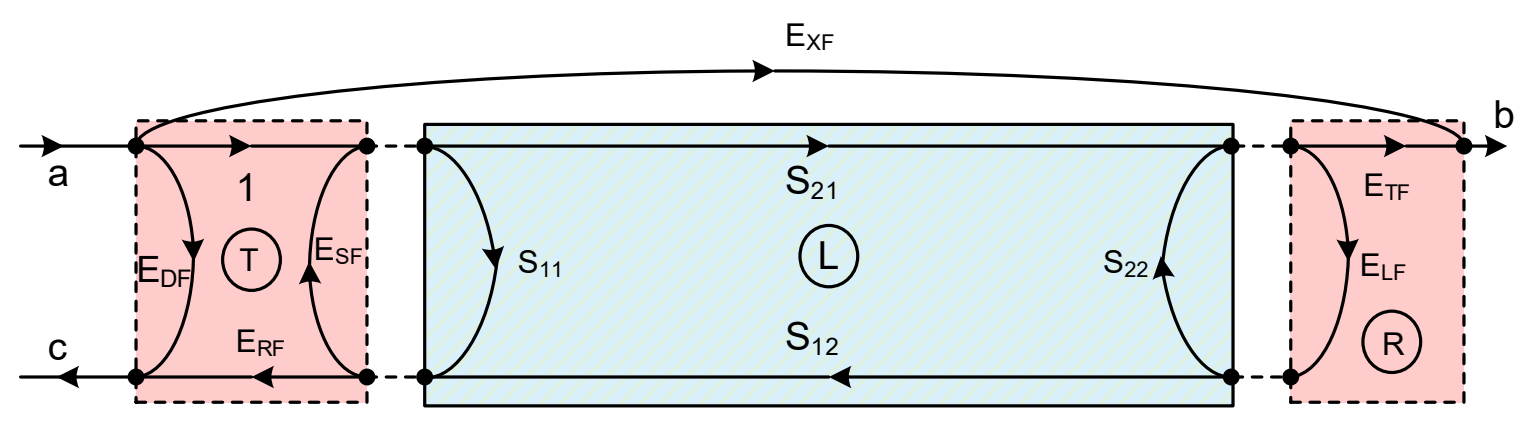

Figure 13: Transmission standard measurement

Path of interest for us is from $a$ to $b$, voltage ratio $b / a=M_{E_{T F}}$ and from $a$ to $c$ ratio $c / a=M_{E_{L F}}$, in this notation "M" means measured value that different from actual. By using the nontouching-loop rule [6] it is possible to find analytically solution for both paths eqs. (32) and (33).

$$
M_{E_{T F}}=\frac{S_{21} E_{T F}}{1-\left(S_{11} E_{S F}+S_{21} E_{L F} S_{12} E_{S F}+E_{L F} S_{22}\right)+S_{11} E_{S F} E_{L F} S_{22}}+E_{X F}
$$




$$
M_{E_{L F}}=\frac{\left(1-E_{L F} S_{22}\right) S_{11} E_{R F}+S_{21} E_{L F} S_{12} E_{R F}}{1-\left(S_{11} E_{S F}+S_{21} E_{L F} S_{12} E_{S F}+E_{L F} S_{22}\right)+S_{11} E_{S F} E_{L F} S_{22}}+E_{D F}
$$

Solution of system $(32,33)$ gives error terms $E_{L F}$ and $E_{T F}$.

$$
\begin{aligned}
& E_{T F}=-\frac{E_{R F} S_{12}\left(E_{X F}-M_{E_{T F}}\right)}{D} \\
& D=E_{D F} E_{S F} S_{11} S_{22}-E_{D F} E_{S F} S_{12} S_{21}-E_{D F} S_{22}-E_{R F} S_{11} S_{22}+ \\
& +E_{R F} S_{12} S_{21}-E_{S F} M_{E_{L F}} S_{11} S_{22}+E_{S F} M_{E_{L F}} S_{12} S_{21}+M_{E_{L F}} S_{22} \\
& E_{L F}=\frac{E_{D F} E_{S F} S_{11}-E_{D F}-E_{R F} S_{11}-E_{S F} M_{E_{L F}} S_{11}+M_{E_{L F}}}{D}
\end{aligned}
$$

\subsection{Cryogenic cables calibration}

Since cables $P_{I}$ and $P_{T}$ are reciprocal devices only one transmission parameter is unknown for each cable $T_{I}$ and $T_{T}$. Reflection parameters $\Gamma_{I W}, \Gamma_{I C}, \Gamma_{T W}, \Gamma_{T C}$ are subject to assumptions discussed above.

To find parameter $T_{I}$ switch 13 is turned in the position " 4 " and cavity is detuned from resonance to achieve full reflection from coupler 21 as it is done in standard procedure [1]. Corresponding graph is shown in fig. 14.

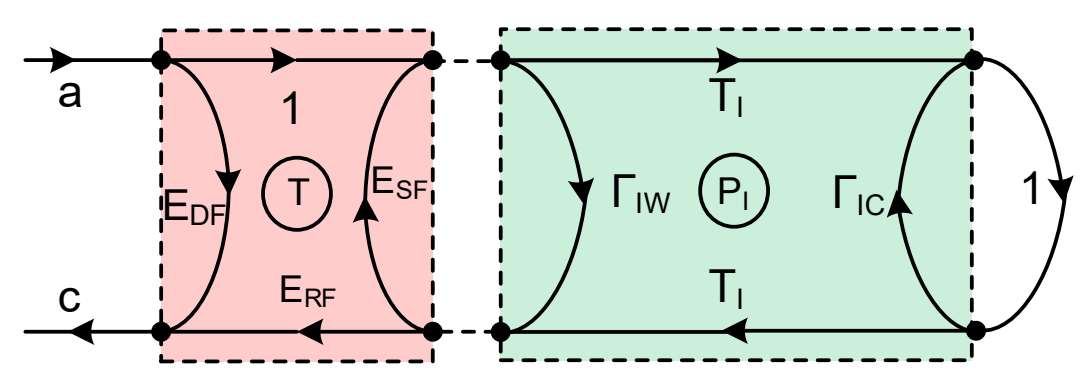

Figure 14: Calibration of $P_{I}$ cable.

In such configuration vector voltage ratio $c / a=M_{T_{I}}$. Using the non-touching loop rule parameter $M_{T_{I}}$ can be defined as eq.(36)

$$
M_{T_{I}}=\frac{\left(1-\Gamma_{I C}\right) \Gamma_{I W} E_{R F}+T_{I}^{2} E_{R F}}{1-\left(\Gamma_{I W} E_{S F}+T_{I}^{2} E_{S F}+\Gamma_{I C}\right)+\left(\Gamma_{I W} E_{S F} \Gamma_{I C}\right)}+E_{D F}
$$

Then from eq.(36) the required $T_{I}$ parameter can be found:

$$
T_{I}=\sqrt{\frac{\left(E_{D F}-M_{T_{I}}\right)\left(\Gamma_{I C}+E_{S F} \Gamma_{I W}-E_{S F} \Gamma_{I C} \Gamma_{I W}-1\right)+E_{R F} \Gamma_{I W}\left(\Gamma_{I C}-1\right)}{E_{R F}-E_{S F}\left(E_{D F}-M_{T_{I}}\right)}}
$$




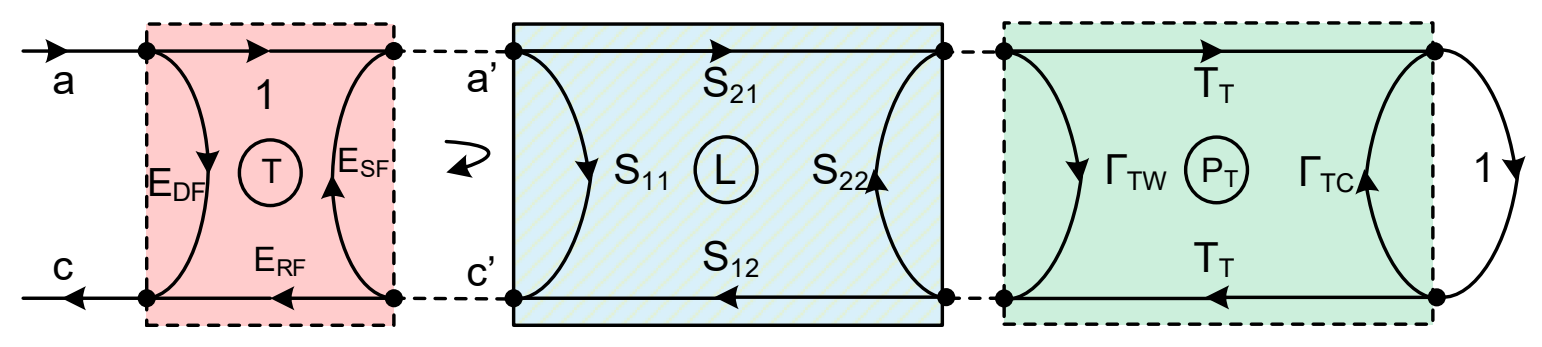

Figure 15: Calibration of $P_{T}$ cable.

Then, to find $T_{T}$ parameter of cable 20 , switch 19 is turned into position "1-2" and switch 13 into position " 5 ". This connects cable 20 to the transmitter "T" through the transmission standard "L" 18. Flow graph is shown in fig.15.

For such graph direct solution $c / a$ using non-touching loop rule is quite bulky, so we will split it in two. First by using the non-touching loop rule we will get reflection from cross-section $a^{\prime}-b^{\prime}$ in form of $M_{T_{T}}^{\prime}$ :

$$
M_{T_{T}}^{\prime}=\frac{\left(1-\Gamma_{T C}\right) S_{21} \Gamma_{T W} S_{12}+S_{21} T_{T}^{2} S_{12}}{1-\left(\Gamma_{T W} S_{22}+T_{T}^{2} S_{22}+\Gamma_{T C}\right)+\Gamma_{T W} S_{22} \Gamma_{T C}}+S_{11}
$$

From eq.(38) $T_{T}$ can be easily extracted as eq.(39) where $M_{T_{T}}^{\prime}$ is unknown:

$$
T_{T}=\sqrt{\frac{\left(M_{T_{T}}^{\prime}-S_{11}\right)\left(\Gamma_{T C}+S_{22} \Gamma_{T W}-S_{22} \Gamma_{T C} \Gamma_{T W}-1\right)+S_{12} S_{21} \Gamma_{T W}\left(\Gamma_{T C}-1\right)}{S_{12} S_{21}+S_{22}\left(M_{T_{T}}^{\prime}-S_{11}\right)}}
$$

And since measured voltage ratio $c / a=M_{T_{T}}$ can be defined as eq. (40),

$$
M_{T_{T}}=E_{D F}+\frac{E_{R F} M_{T_{T}}^{\prime}}{1-E_{S F} M_{T_{T}}^{\prime}}
$$

the unknown value of $M_{T_{T}}^{\prime}$ for eq. (39) can be found from (40) as:

$$
M_{T_{T}}^{\prime}=\frac{M_{T_{T}}-E_{D F}}{E_{R F}-E_{D F} E_{S F}+E_{S F} M_{T_{T}}}
$$

For a perfect match assumption in the $P_{I}$ and $P_{T}$ cable connectors $\Gamma_{I W}=\Gamma_{I C}=$ $\Gamma_{T W}=\Gamma_{T C}=0$ so eq.(37) and eq.(39) will reduce to eq.(42) and eq.(43) correspondingly.

$$
T_{I}=\sqrt{\frac{M_{T_{I}}-E_{D F}}{E_{R F}-E_{D F} E_{S F}+E_{S F} M_{T_{I}}}}
$$

If $P_{t}$ cable match is perfect on both ends reduces to

$$
T_{T}=\sqrt{\frac{M_{T_{T}}^{\prime}-S_{11}}{S_{12} S_{21}+S_{22}\left(M_{T_{T}}^{\prime}-S_{11}\right)}}
$$


In order to reduce additional complexity in the equations, the above calibration procedures do not include switch terms like it is done in [12] and we assume equal attenuation and electrical lengths of paths in SP5T switch 13 and matrix switch 19 which for our frequency range $0.1-10 \mathrm{GHz}$ can be easily achieved in practice.

\subsection{Summary on vector calibration and cavity measurements}

Below we summarize algorithm of VTS vector calibration and $Q_{0}\left(E_{a c c}\right)$ measurement.

I. Calibration. Detune cavity from resonance.

1) Turn switch 13 in position "1". Measure $L_{M}=c / a$, measure port power $P_{\text {port }}$ with power meter 15 directly.

2) Turn switch 19 in position "3-2". Measure $E_{X F}=b / a$;

3) Turn switch 13 in position " 2 ". Measure $O_{M}=c / a$;

4) Turn switch 13 in position "3". Measure $S_{M}=c / a$;

5) Find $E_{D F}, E_{R F}, E_{S F}$ using eqs. (29), (30), (31);

6) Turn switch 13 in position "5", switch 19 in position "1-3". Measure $M_{E_{T F}}=b / a$ and $M_{E_{L F}}=c / a$;

7) Find $E_{T F}, E_{L F}$ using eqs. (34), (35);

8) Turn switch 19 in position "1-2". Measure $M_{T_{T}}=c / a$;

9) Find $M_{T_{T}}^{\prime}$ using eq.(41), then find $T_{T}$ with eq. (43);

10) Turn switch 13 in position " 4 ". Measure $M_{T_{I}}=c / a$;

11) Find $T_{I}$ using eq. (42).

II. Cavity measurement. Tune cavity to resonance, lock PLL.

Switch 13 should be in position "4", switch 19 in position "3-2". First, loaded quality factor $Q_{L}$ of the cavity is measured using standard decay technique. After that:

1) Measure $M_{T}=b / a, M_{\Gamma}=c / a$;

2) Find cavity transmission $T$ and reflection $\Gamma$ using eqs. $(25,24)$

3) Determine coupling $C_{\beta}$ of the cavity from the phase $\phi$ of the reflection coefficient $\Gamma$. For overcoupled cavity phase will be somewhere in ranges $\left(0^{\circ}<\phi<90^{\circ}\right)$ and $\left(270^{\circ}<\phi<360^{\circ}\right)$. For undercoupled cavity phase will be in range $\left(90^{\circ}<\phi<270^{\circ}\right)$;

3) Find $Q_{0}$ using eq. (44) (by analogy with eq. (11);

$$
Q_{0}=\frac{2 Q_{L}\left(C_{\beta}|\Gamma|-1\right)}{|\Gamma|^{2}+|T|^{2}-1}
$$

4) Find power $P_{i}$ at the input cavity coupler 21 simply by applying attenuation $T_{I}$ of the cable 17 to the measured earlier port power:

$$
P_{i}=P_{\text {port }}\left|T_{I}\right|^{2}
$$

5) Find accelerating gradient $E_{a c c}$ using eq.(46), see eqs. $(2,9)$ for reference.

$$
E_{a c c}=\kappa \sqrt{Q_{0}\left(P_{i}-P_{i}\left|\Gamma_{M}\right|^{2}-P_{i}\left|T_{M}\right|^{2}\right)}
$$




\section{Final Comments}

The proposed above vector calibration procedure and hardware has several advantages over the techniques currently used for vertical cavity tests:

1) It provides high level of automation and reduces manpower and preparation time for the test.

2) It provides in situ, real time control of VTS hardware parameters and allows to detect hardware failure and operator's errors.

3) It allows to control and exclude the most significant systematic uncertainties, measured values of $Q_{0}\left(E_{a c c}\right)$ can be made traceable to national standards.

4) For sub-Kelvin experiments, eq.(44) with setup from [10] can be used for the direct measurement of $Q_{0}$ in the dilution refrigerator, without necessity to preliminary measure cavity coupling in VTS.

Several aspects, however, remain unfinished: cryogenic cable mismatches are not characterized in the proposed procedure and assumed to be negligibly small. For extra high-accuracy, such characterization requires moving calibration standards in cryogenic environment, which remains unsolved problem for $\mathrm{kW}$-range power levels used for accelerating cavities' tests. Or different methods of calibration such as time-domain reflectomerty should be used. These problems are subject of our ongoing research. 


\section{References}

[1] Tom Powers, Theory and Practice of Cavity RF Test Systems, Proceedings of the $12^{\text {th }}$ International Workshop on RF Superconductivity, Cornell University, Ithaca, New York, 2005

[2] W. Schappert, et al. Systematic Uncertainties in RF-Based Measurement of Superconducting Cavity Quality Factors, Proceedings of SRF2015, Whistler, Canada, 2015, pp. 814-818

[3] Vitaliy A. Goryashko, et al A Method for High-Precision Characterization of the Q-Slope of Superconducting RF Cavities, IEEE Transactions on Microwave Theory and Techniques, Vol. 64, No. 11 November, 2016

[4] O. Melnychuk, A. Grassellino and A. Romanenko Error Analysis of Intrinsic Quality Factor Measurement in Superconducting Radio Frequency Resonators, Review of Scientific Instruments 85, 124705 (2014)

[5] Cletus A. Hoer A Network Analyzer Incorporating Two Six-Port Reflectometers, IEEE Transactions on Microwave Theory and Techniques, Vol. MTT-25, No. 12 December, 1977

[6] Stephen F. Adam Microwave Theory And Applications, Hewlett-Packard, 1969, p.105

[7] Robert A. Witte Spectrum and Network Measurements, Second Edition by SciTech Publishing, 2014, p.289

[8] Joel P. Dunsmore Handbook of Microwave Measurements: with advanced VNA techniques, John Wiley \& Sons, 2012, p.126

[9] Michael Hiebel Fundamentals of Vector Network Analysis, Rohde \& Schwarz, Fifth edition 2011, p.114

[10] Leonardo Ranzani et al. Two-port microwave calibration at millikelvin temperatures, Review of Scientific Instruments 84, 034704 (2013)

[11] W. Kruppa, K.F. Sodomsky An Explicit Solution for the Scattering Parameters of a Linear Two-Port Measured with an Imperfect Test Set, IEEE Transactions on Microwave Theory and Techniques, Vol. 19, Issue 1 January, 1971

[12] Roger B. Marks Formulations of the Basic Vector Network Analyzer Error Model including Switch Terms, 50th ARFTG Conference Digest, 4-5 December, Portland, Oregon, 1997 